\title{
Effect of PNF, ballistic and static stretching on the range of motion after sports injury surgery in football athletes
}

\author{
Heidar Sajedi ${ }^{1 \star}$, Metin Bayram and Murat Bilgiç ${ }^{2}$ \\ ${ }^{1}$ Department of Physical Education, Ağrı İbrahim Çeçen University, Ağrı, Turkey. \\ ${ }^{2}$ School of Physical Education and Sports, Batman University, Turkey.
}

Accepted 24 March, 2020

\begin{abstract}
The purpose of this study was to investigate the effect of proprioceptive neuromuscular facilitation (PNF), ballistic, and static stretching exercises on the range of motion after sports injury surgery in soccer team athletes. The purpose of this research was an applied one, in terms of method was descriptive and correlational one. Therefore, in terms of data collection, the research method was the laboratory strategy. The statistical population of this study was injured male students of soccer players who had undergone surgery. The sample included 97 students (30 active ballistic, 30 active statics and 37 PNF stretching exercises). Statistical analysis of the data was done by SPSS 20 software and t-test. Overall, the results showed that PNF stretching had a significant effect, statics stretching had moderate impact and ballistic stretching had a low but significant effect on the range of motion after sports injury in soccer athletes. That is, PNF had the best effect in terms of increasing the range of motion of the muscles after surgery, followed by static and ballistic stretching, respectively.
\end{abstract}

Keywords: PNF exercises, ballistic exercises, static exercises, muscle range, sports injury surgery.

E-mail: sajedy_haeidar@yahoo.com.

\section{INTRODUCTION}

The importance of flexibility in various researches is an important factor in the rehabilitation, injury prevention and performance improvement in athletes (Cayco et al., 2016). Some research suggests accelerating the process of recovery and rehabilitation of athletes in the post-injury period. These studies have each reported some kind of flexibility development due to the regular implementation of stretching programs (Weng et al., 2009).

The PNF method is a tensile technique first used by Kabat to treat paralysis patients and then used by other researchers to increase flexibility and strength in diverse groups of athletes (Yuktasir and Kaya, 2009). Holt, Travis, Okita, Sadie, Wertman, Blank, and Toni Gawa observed a greater increase in flexibility by combining PNF techniques with active (ballistic and static) techniques (Khamwong et al., 2011).

The term PNF stands for Proprioceptive Neuromuscular Facilitation (Ferber et al., 2002). PNF stretching is the method of accelerating or expanding the neuromuscular mechanism by stimulating deep receptors that can relax the muscle. This type of stretching is known to be the fastest and most effective method of improving static and passive flexibility (Krukowska et al., 2016).

This type of stretch is a combination of passive stretching and isometric stretching to achieve maximum static flexibility. Most PNF tensile movements agree with isometric contraction of the muscles (Espejo-Antúnez et al., 2016). However, some PNF stretching techniques are performed with the contraction of the opposing muscles that have been stretched (Feland et al., 2001). In all of these, the essential thing is to stretch the muscle for 20 seconds before the next stretch and relax. The most common PNF stretching methods are a combination of agonist and antagonist contraction or stretching and relaxation (Khodayari and Dehghani, 2012).

In ballistic flexibility techniques, the torque of the body and limbs is used to move a member beyond its usual 
range of motion (Arceo et al., 2018). Some believe that this type of stretching is not a good way to improve flexibility and it causes injury (Kubo et al., 2018). Launching tensile movements do not allow the muscle to relax and stretch in position, and the continuous calling of the Stretch Reflex results in muscle stiffness (Ceyhun and Makoc, 2015).

Ballistic stretching, also known as active stretching, uses movement and propulsion to stretch muscle. Ballistic stretching involves a series of rapid movements that become a specific stretching condition (Giroux et al., 2015). In this method, sequential contractions of agonist muscles are used to induce rapid antagonist muscle stretches. Active contraction and muscle stretching is done using gravity. If the force produced by jerking is greater than the stretching of the muscle, it may cause muscle damage. Consistent and severe contractions of agonist muscle that lead to antagonist muscle tension can also cause muscle pain (Nishimoto and Takasaki, 2019).

In general, in this technique, repeated agonist muscle contractions are used to elicit antagonist muscles rapidly (De Souza et al., 2016). The safety of these types of stretches has not been particularly proven for residents and athletes with muscle damage (Taniguchi et al., 2015). Given the application of ballistic stretching in daily exercise and the risks associated with this movement, they should be the final stages of healing in the rehabilitation program (Riley and Van Dyke, 2012).

Active static flexibility techniques also include stretching the member at the background and holding it for a few seconds (Umegaki et al., 2015), such as fully opening two legs apart (Smajla et al., 2019). Trainers and athletes commonly consider static stretching with passive stretching to be meaningful (Fortney et al., 2015) and to use interchangeably (Nojiri et al., 2019), whereas static and passive stretching differs.

Inactive stretching exercise is performed when the muscle is relaxed with the help of another person or a specific device (Ceyhun and Makoc, 2017), but static stretching is performed on the limb to the maximum extent of movement by the individual (Kubo, 2018).

Static stretching slowly becomes a stretching condition (Campos de Oliveira et al., 2016) and the individual may maintain a stretching position for 10 to 30 seconds (Kimoto et al., 2015). More static stretching to cool after exercise are appropriate (Wanderley et al., 2019). However, one can also use static light stretching to warm up (Turgut and Mtlu yasar, 2019). After cooling while the muscles are still warm, one can do more stretching (Sharma et al., 2015). A good stretch can be very relaxing by releasing the mild contractions that occur in the muscles throughout the day (Salazar et al., 2018).

On the other hand, athletic injuries and surgeries that occur after these injuries to athletes are those in which the range of motion and flexibility of the muscles is increased (Espejo-Antúnez et al., 2016). And the use of PNF techniques along with active flexibility techniques such as ballistic and static techniques can work (Abbasi et al., 2019). Soccer is one of the high-risk sports that have different muscular injuries. Injured soccer athletes experience reduced flexibility and range of motion after surgery to investigate the effect of PNF, ballistic and static stretching exercises on the range of motion after surgery. Sports Injuries to soccer athletes should be addressed and determined to what extent each of these techniques can increase and improve the range of motion of the muscles.

\section{METHODOLOGY}

The purpose of this study was applied research, in terms of method was descriptive and correlational. Therefore, in terms of data collection, the research method is the laboratory strategy. The statistical population of this study was injured male soccer players who were operated on. For two years, the researcher studied traumatized soccer athletes who had undergone surgery at Mohaghegh Ardabili University Health and Wellness Center. After two months of physiotherapy that involved flexibility and strength training that results in muscle stiffness and muscle loss, PNF and active stretching (including ballistic and static exercises) for two months were done on subjects.

During this period, 97 men in the age range of $19-30$ years and height of 170 to $195 \mathrm{~cm}$ participated in the study. Statistical sample included 30 athletes in active ballistic, 30 in static, as well as 37 in PNF stretching exercises. In this study, the Wells test was used to evaluate lumbar and hamstring flexibility, and goniometer was used to examine the flexibility and range of motion of the knee and ankle joints. So pre and post tests were taken at the start and after two months of flexibility exercises. All the injured athletes were from the field of soccer and their injuries were from the knee and ankle. In this study, the range of motion of the knee and ankle joints and hamstring flexibility were measured. Statistical analysis of the research data was done using SPSS 20 software and t-test.

\section{RESULTS}

\section{Effects of PNF stretching exercises on the range of motion after sports injury in football athletes}

According to the results of Table 1 and considering that the significance level of the test error less than 0.01 , it can be concluded that PNF stretching exercises had a significant effect on the range of motion after sports injury surgery in soccer athletes. As the averages show, the mean range of motion after PNF stretching (20.837) increased by 10.216 compared to the mean range of motion before PNF stretching (10.621), which was also due to the observed difference. 
Table 1. Results of the statistical test of "two-way dependent groups (Paired)" to investigate the effect of PNF stretching exercises on range of motion.

\begin{tabular}{cccccc}
\hline $\begin{array}{c}\text { The average range of } \\
\text { motion before the PNF }\end{array}$ & $\begin{array}{c}\text { The average range of } \\
\text { motion after the PNF }\end{array}$ & Average difference & $\mathbf{T}$ & df & Sig. (2-tailed) \\
\hline 10.621 & 20.837 & 10.216 & 11.708 & 36 & 0.000 \\
\hline
\end{tabular}

Table 2. Results of the "two-tiered dependent t-test" statistical test to evaluate the impact of ballistic stretching exercises on the range of motion.

\begin{tabular}{cccccc}
\hline $\begin{array}{c}\text { The average range of } \\
\text { motion after the Ballistic }\end{array}$ & $\begin{array}{c}\text { The average range of } \\
\text { motion before the Ballistic }\end{array}$ & Average difference & $\mathbf{T}$ & df & Sig. (2-tailed) \\
\hline 10.866 & 10.633 & 0.233 & 2.536 & 29 & 0.017 \\
\hline
\end{tabular}

Table 3. Results of the "two-tailed dependent t-test" for the study of the effect of static stretching exercises on the range of motion.

\begin{tabular}{cccccc}
\hline $\begin{array}{c}\text { The average range of } \\
\text { motion after the static }\end{array}$ & $\begin{array}{c}\text { The average range of } \\
\text { motion before the static }\end{array}$ & Average difference & $\mathbf{T}$ & df & Sig. (2-tailed) \\
\hline 14.367 & 10.624 & 3.743 & 6.211 & 29 & 0.003 \\
\hline
\end{tabular}

\section{Effect of ballistic stretching exercises on range of motion after sports injury surgery in soccer athletes}

According to the results of Table 2 and considering that the significance level of test error less than 0.05 , it can be concluded that ballistic stretching exercises had a significant effect on the range of motion after sports injury surgery in soccer team athletes. As the averages show, the mean range of motion after ballistic stretching (10.866) increased by 0.233 compared to the mean range of motion before ballistic stretching (10.633), which was also due to the observed difference.

\section{Evaluation of the effect of static stretching exercises on the range of motion after sports injury surgery in athlete football students}

According to the results of Table 3 and considering that the significance level of test error less than 0.01, therefore, it can be concluded that static stretching exercises had a significant effect on the range of motion after sports injury surgery in soccer team athletes. As the averages show, the mean range of motion after static stretching exercises (14.367) increased by 3.743 compared to the mean range of motion before static stretching exercises (10.624), which was also due to the observed difference.

\section{DISCUSSION AND CONCLUSION}

The purpose of this study was to investigate the effect of PNF stretching and ballistic and static stretching exercises on range of motion after sports injury surgery in soccer athletes.

Regarding the effect of PNF stretching exercises on the range of motion after sports injury surgery in football athletes, the results showed that: PNF stretching exercises had a significant effect on the range of motion after sports injury surgery in football athletes. As the averages also showed, the mean range of motion after PNF stretching (20.837) was 10.216 compared to the mean range of motion prior to PNF stretching (10.621), which was also due to the observed difference. It can be concluded that PNF stretch training significantly (10.216) significantly increased the range of motion after sports injury surgery in soccer athletes.

Concerning the results of this section, it can be concluded that the use of the acceleration or development of the neuromuscular mechanism through the stimulation of deep receptors that induce relaxation of the muscle has been able to increase the range of motion of the muscles after surgery of athletes.

The results of this section are in line with the studies of Ceyhun and Makoc $(2015,2017)$, Khamwong et al. (2011), Krukowska et al. (2016), Espejo-Antúnez et al. (2016) and Ferber et al. (2002).

Concerning the effect of ballistic stretching exercises on the range of motion after sports injury surgery in football athletes, the results showed that the following; ballistic stretching exercises on the range of motion after sports injury surgery in football athletes. As the averages show, the mean range of motion after ballistic stretching (10.866) increased by 0.233 compared to the mean range of motion before ballistic stretching (10.633), which was also due to the observed difference.

It can be concluded that ballistic stretching training low 
significantly increased the range of motion (0.233) after sports injury surgery in soccer athletes.

Concerning the results of this section, it can be concluded that using the torque method of moving the body to a range beyond its usual range of motion as well as the use of throwing throws can also be harmful if careless. Also, it was able to significantly increase the range of motion of the post-operative muscle injuries in soccer athletes.

The results of this section are in line with the research of Kubo et al. (2018), Ceyhun and Makoc (2017), Giroux et al. (2015), Arceo et al. (2018) as well as Nishimoto and Takasaki (2019).

Regarding the effect of static stretching exercises on the range of motion after sports injury surgery among soccer players, the results showed that; static stretching exercises had significance effect on the range of motion after sports injury surgery. As the averages show, the mean range of motion after static stretching exercises (14.367) increased by 3.743 compared to the mean range of motion before static stretching exercises (10.624), which was also due to the observed difference. It can be concluded that static stretching exercises significantly (0.233) increased the range of motion after sports injury surgery in college football athletes.

Concerning the results of this section, it can be concluded that the use of stretching the limb in maximum and holding it for a few seconds has been able to increase in moderate the range of motion of the muscles after sports injuries among football athletes.

The results of this section are consistent with the research by Smajala et al. (2019), Nojiri et al. (2019), Umegaki et al. (2015), Kruse et al. (2015), Kimoto et al. (2015), Sharma et al. (2015), Salazar et al. (2018).

As a general conclusion, it can be said that the PNF training (stretching and holding it for a few seconds) increased the range of motion of the muscles more than the other two stretching methods (ballistic and static stretching exercises) after surgery. In addition with comparison of static and ballistic stretching method, the static method increased the range of motion of the muscles more than the other one. That is, PNF had the best effect in terms of increasing the range of motion of the muscles after surgery, followed by static and ballistic stretching, respectively.

\section{REFERENCES}

Abbasi, A., Zamanian, M., and Svoboda, Z. (2019). Nonlinear approach to study the acute effects of static and dynamic stretching on local dynamic stability in lower extremity joint kinematics and muscular activity during pedalling. Human Movement Science, 66: 440-448.

Arceo, S. R., Runner, R. P., Huynh, T. D., Gottschalk, M. B., and Moore, T. J. (2018). Disparities in follow-up care for ballistic and nonballistic long bone lower extremity fractures. Injury, 49(12): 21932197.

Campos de Oliveira, L., Gonçalves de Oliveira, R., and Pires-Oliveira, D. A. (2016). Comparison between static stretching and the Pilates method on the flexibility of older women. Journal of Bodywork and
Movement Therapies, 20(4): 800-806.

Cayco, C., Labro, A., and Gorgon, E. J. (2016). Effects of proprioceptive neuromuscular facilitation on hamstrings flexibility in adults: a systematic review with meta-analysis. Physiotherapy, 102(Supplement 1): e190.

Ceyhun, S., Makoc, N. (2015). Communication skills levels of Karabuk UniversitySchoolof Physical Education and Sports students, International Journal of Social Sciences and Educational Research, 1(4): 1297- 1304.

Ceyhun, S., and Makoc, N. (2017). Investigation of general cynicism level of personnel working in national sports special sports enterprises. National Journal of Sports Sciences, 1(2): 12-26.

Turgut, M., and Mutlu Yasa, O. (2019). Playing digital game motivations of university students. Asian Journal of Education and Training, 5(4): 603-608.

Ceyhun, S., and Makoc, N. (2015). Communication skills levels of Karabuk University School of Physical Education and Sports students, International Journal of Social Sciences and Educational Research, 1(4): 1297- 1304.

De Souza, L. M. L, Paz, G. A., Eloi, I. L., Dias, R., and Lima, V. P. (2016). Vertical jump performance after passive static stretching of knee flexors muscles. Apunts. Medicina de l'Esport, 51(192): 131136.

Espejo-Antúnez, L., Castro-Valenzuela, E., Ribeiro, F., AlbornozCabello, M., Rodríguez-Mansilla, J. (2016). Immediate effects of hamstring stretching alone or combined with ischemic compression of the masseter muscle on hamstrings extensibility, active mouth opening and pain in athletes with temporomandibular dysfunction. Journal of Bodywork and Movement Therapies, 20(3): 579-587.

Espejo-Antúnez, L., López-Miñarro, P. A., Albornoz-Cabello, M., Garrido-Ardila, E. M. (2016). Acute effect of electrical muscle elongation and static stretching in hamstring muscle extensibility. Science and Sports, 31(1): e1-e7.

Feland, J. B., Myrer, J. W., and Merrill, R. M. (2001). Acute changes in hamstring flexibility: PNF versus static stretch in senior athletes. Physical Therapy in Sport, 2(4): 186-193.

Ferber R., Osternig, L. R., and Gravelle, D. C. (2002). Effect of PNF stretch techniques on knee flexor muscle EMG activity in older adults. Journal of Electromyography and Kinesiology, 12(5): 391-397.

Fortney, B. P., McReynolds, L. E., Muse, T. J., Neelly, C. T., and Hoover, D. L. (2015). Neither dynamic warm-up nor static stretching affect measures of power and fatigue during vigorous cycling among women. Physiotherapy, 101(Supplement 1): e400.

Giroux, C., Guilhem, G., Couturier, A., Chollet, D., and Rabita, G. (2015). Is muscle coordination affected by loading condition in ballistic movements? Journal of Electromyography and Kinesiology, 25(1): 69-76.

Khamwong, P., Pirunsan, U., and Paungmali, A. (2011). A prophylactic effect of proprioceptive neuromuscular facilitation (PNF) stretching on symptoms of muscle damage induced by eccentric exercise of the wrist extensors. Journal of Bodywork and Movement Therapies, 15(4): 507-516.

Khodayari, B., and Dehghani, Y. (2012). The investigation of mid-term effect of different intensity of PNF stretching on improve hamstring flexibility. Procedia - Social and Behavioral Sciences, 46: 5741-5744.

Kimoto, Y., Wakasa, M., Shuit, C., Nakazawa, A., and Satake, M. (2015). Acute effects of static stretching and dynamic stretching on range of motion and isometric muscle strength of the quadriceps, Physiotherapy, 101(1): e753-e754.

Krukowska, J., Bugajski, M., Sienkiewicz, M., and Czernicki, J. (2016). The influence of NDT-Bobath and PNF methods on the field support and total path length measure foot pressure (COP) in patients after stroke. Neurologia i Neurochirurgia Polska, 50(6): 449-454.

Kruse, N. T., Barr, M. W., Gilders, R. M., Kushnick, M. R., and Rana, S. R. (2015). Effect of different stretching strategies on the kinetics of vertical jumping in female volleyball athletes. Journal of Sport and Health Science, 4(4): 364-370.

Kubo, K. (2018). Effects of static stretching on mechanical properties and collagen fiber orientation of the Achilles tendon in vivo. Clinical Biomechanics, 60: 115-120.

Kubo, K., Ishigaki, T., and Ikebukuro, T. (2018). Measurement of active muscle stiffness with and without the stretch reflex. Journal of 
Biomechanics, 73: 50-59.

Nishimoto, K., and Takasaki, H. (2019). Jack-knife stretching and active knee extension stretching equally improve the relative flexibility of the hamstring muscles between the low back: A randomized controlled trial. Physical Therapy in Sport, 38: 139-145.

Nojiri, S., Ikezoe, T., Nakao, S., Umehara, J., and Ichihashi, N. (2019). Effect of static stretching with different rest intervals on muscle stiffness. Journal of Biomechanics, 90: 128-132

Riley, D. A., and Van Dyke, J. M. (2012). The effects of active and passive stretching on muscle length. Physical Medicine and Rehabilitation Clinics of North America, 23(1): 51-57.

Salazar, A. P., Pinto, C., Mossi, J. V. R., Figueiro, B., and Pagnussat, A. S (2018). Effectiveness of static stretching positioning on poststroke upper-limb spasticity and mobility: Systematic review with meta-analysis. Annals of Physical and Rehabilitation Medicine, 62(4): 274-282.

Sharma, S., Balthillaya, G., Danait, R., and Mani, R. (2015). Effectiveness of neural sliders and neural tensioners as an adjunct to static stretching on hamstrings flexibility: a randomized controlled trial. Physiotherapy, Volume 101, Supplement 1.

Smajla, D., García-Ramos, A., Tomazin, K., and Strojnik, V. (2019). Selective effect of static stretching, concentric contractions, and a one-leg balance task on ankle motion sense in young and older adults. Gait and Posture, 71: 1-6.

Taniguchi, K., Nozaki, S., and Katayose, M. (2015). Resting muscle stiffness measured with ultrasound shear-wave elastography after static stretching. Physiotherapy, 101(Supplement 1): e1489.

Umegaki, H., Ikezoe, T., Nakamura, M., Nishishita, S., and Ichihashi, N. (2015). Acute effects of static stretching on the hamstrings using shear elastic modulus determined by ultrasound shear wave elastography: Differences in flexibility between hamstring muscle components. Manual Therapy, 20(4): 610-613.

Wanderley, D., Valença, M. M., Neto, J. J. C., Martins, J. V., de Oliveira, D A. (2019). Contract-relax technique compared to static stretching in treating migraine in women: A randomized pilot trial. Journal of Bodywork and Movement Therapies, In press, corrected proof, Available online 22 May 2019.
Weng, M. C., Lee, C. L., Chen, C. H., Hsu, J. J., Lee, W. D., Huang, M. $\mathrm{H}$., and Chen, T. W. (2009). Effects of different stretching techniques on the outcomes of isokinetic exercise in patients with knee osteoarthritis. The Kaohsiung Journal of Medical Sciences, 25(6): 306-315.

Yuktasir, B., and Kaya, F. (2009). Investigation into the long-term effects of static and PNF stretching exercises on range of motion and jump performance. Journal of Bodywork and Movement Therapies, 13(1): 11-21.

Citation: Sajedi, H., Bayram, M., and Bilgiç, M. (2020). Effect of PNF, ballistic and static stretching on the range of motion after sports injury surgery in football athletes. African Educational Research Journal, 8(1): 105-109. 